\title{
Optical properties of cosmic dust analogs: A review
}

\author{
Thomas Henning ${ }^{\mathrm{a}}$ and Harald Mutschke ${ }^{\mathrm{b}}$ \\ àax-Planck-Institut für Astronomie, Königstuhl 17, D-69117 Heidelberg \\ henning@ mpia-hd.mpg.de \\ ${ }^{\mathrm{b}}$ Astrophysical Institute and University Observatory (AIU), Friedrich Schiller University, \\ Schillergäßchen 3, D-07745 Jena, Germany \\ mutschke@astro.uni-jena.de
}

\begin{abstract}
Nanometer- and micrometer-sized solid particles play an important role in the evolutionary cycle of stars and interstellar matter. The optical properties of cosmic grains determine the interaction of the radiation field with the solids, thereby regulating the temperature structure and spectral appearance of dusty regions. Radiation pressure on dust grains and their collisions with the gas atoms and molecules can drive powerful winds. The analysis of observed spectral features, especially in the infrared wavelength range, provides important information on grain size, composition and structure as well as temperature and spatial distribution of the material.

The relevant optical data for interstellar, circumstellar, and protoplanetary grains can be obtained by measurements on cosmic dust analogs in the laboratory or can be calculated from grain models based on optical constants. Both approaches have made progress in the last years, triggered by the need to interpret increasingly detailed high-quality astronomical observations. The statistical theoretical approach, spectroscopic experiments at variable temperature and absorption spectroscopy of aerosol particulates play an important role for the successful application of the data in dust astrophysics.
\end{abstract}

Keywords: cosmic dust, optical constants, infrared spectroscopy, laboratory astrophysics

\section{INTRODUCTION}

Dust grains exist in a wide variety of cosmic environments ranging from the diffuse interstellar medium and molecular clouds in our galaxy to circumstellar shells and disks around young stellar objects, dusty outflows from evolved stars, atmospheres of brown dwarfs, the dust tori of active galactic nuclei and starburst galaxies, and high-redshift quasars. The particles are especially important during the birth process of stars and as precursor material for the formation of planets. In the late stages of stellar evolution they condense in molecular outflows and are important for the mass loss process and the spectral appearance of AGB and post-AGB stars.

The field of cosmic-dust research has been the subject of intense research over the last decades. The international conferences "Astrophysics of Dust" in Estes Park, Colorado (2003) and "Cosmic Dust - Near and Far" in Heidelberg, Germany (2008) both reflected the rapid development of the field. Astronomical dust research is of course triggered by the progress in astronomical observations, mainly by those in the infrared range, because of the opportunities to (1) observe dusty objects optically thick in the visible and (2) to analyse the thermal emission of dust by infrared spectroscopy. The ISO and Spitzer space telescopes have played a major role in this respect, and current and future projects such as the Herschel mission and JWST will continue to ensure progress in this field.

The description of the interaction of radiation fields with a system of solid particles always requires knowledge of their absorption and scattering cross sections which depend on their chemical composition, solid-state structure and morphology (see Table 1). Any continuum radiative transfer code for the calculation of intensity/polarization maps and spectral energy distributions needs the specification of these properties. In addition, the transfer of momentum 
Table 1. Chemical and structural properties of solid particles and the description of optical properties on different length scales

Length scale Structural properties Description

$\begin{array}{lll}\text { "Atomic" } & \begin{array}{l}\text { Chemical composition and bonding } \\ \text { Crystal structure } \\ \text { Defects and impurities }\end{array} & \begin{array}{l}\text { Dielectric function } \epsilon(\lambda) \\ \text { Complex refractive index } m(\lambda)\end{array} \\ \text { "Mesoscopic" } & \begin{array}{l}\text { Inhomogeneities } \\ \text { Porosity } \\ \text { Mantles } \\ \text { Surface states }\end{array} & \begin{array}{l}\text { Theory of effective media } \\ \epsilon_{\text {eff }}(\lambda), \\ \text { Core-mantle description }\end{array} \\ \text { "Macroscopic", } & \begin{array}{l}\text { Size distribution } \\ \text { Grain shapes }\end{array} & \\ \text { Morphology } & \begin{array}{l}\text { Agglomeration } \\ \text { Coalescence }\end{array} & \begin{array}{l}\text { Mie theory, T-matrix method, } \\ \text { Separation of variables method, } \\ \text { Discrete dipole approximation } \\ C_{\text {ext }}(\lambda), C_{\text {abs }}(\lambda), C_{\text {sca }}(\lambda)\end{array}\end{array}$

from a radiation field to a grain is determined by the extinction and scattering efficiency and the average cosine of the scattering angle.

In this review we will deal with the progress in theoretical and experimental work aimed to providing data and tools for the interpretation of spectroscopic dust-related observations, and for modeling of dusty astrophysical objects. Current models go significantly beyond the assumption of spherical grains characterized by artificially constructed optical constants, as has been the prefered approach for many years. Effects of grain morphology, temperature, and composition have been studied experimentally, and complex light-scattering models have been used to explore the dependence of scattering and absorption cross sections on the structure of grains on all length scales (see Table 1, including porosity, inhomogeneity, and agglomeration. Such grain models have been used in sophisticated multi-dimensional radiative transfer codes. Among the experimental data, which can be included as inputs into the computations, are also measurements of the scattering matrices of cosmic dust analogs which have been performed in Amsterdam for many years and have been made available through the Amsterdam Light Scattering Database (http://www.astro.uva.nl/scatter). Although this review unfortunately cannot cover this and some other experimental fields devoted to interpret observational data of cosmic dust, all general considerations presented in the next sections are valid for them as well, especially those on the relation between calculations and measurements.

It is beyond the scope of this paper to discuss extensively the astronomical dust models and optical data derived from observations. For a description of the various "classical" dust models, we refer to the contributions in Ref. 1. More recent models have been developed by Mathis [2], Li \& Greenberg [3], Zubko et al. [4], Draine \& Li [5] and can also be found in the proceedings of the two dust conferences [6,7].

\section{TWO WAYS FOR PREDICTING OPTICAL DATA OF COSMIC DUST PARTICLES}

Our knowledge about the nature of cosmic dust grains mainly comes from spectroscopic observations over the infrared, optical, and UV wavelength ranges. Infrared spectroscopy provides information about vibrational modes of functional groups and crystal lattices, whereas the optical and UV range of the galactic extinction curve is mainly dominated by a strong UV resonance 




Fig. 1. Two ways for determining optical properties of cosmic dust analogs.

at $217 \mathrm{~nm}$, usually attributed to an electronic transition in carbonaceous grains or polyaromatic hydrocarbons (PAHs). In the infrared, features related to the abundant silicates are frequently observed, especially the bands around 10 and $18 \mu \mathrm{m}$ attributed to Si-O stretching and bending vibrations.

Other constraints for the chemical and physical structure of grains are provided by abundance considerations, by our understanding of the temperature and density range, as well as by the presence of shocks and winds in interstellar and circumstellar media (see, e.g., Refs. 8 and 9). In oxygen-rich environments we can expect the presence of oxide materials (mainly silicates) and oxygen-containing ices $\left(\mathrm{H}_{2} \mathrm{O}, \mathrm{CO}_{2}\right)$. Carbon-rich environments contain mainly carbonceous solids, carbides, and sulfides. Here, we will only deal with refractory materials and not with molecular ices [10,11] and larger molecules such as PAHs [12,13].

After the discovery of abundant crystalline minerals in circumstellar disks (e.g., Refs. 14 and 15), and the increasing efforts to study the detailed mineralogy and morphology of the dust in these environments from its infrared emission spectra, both theorists working on lightscattering theory and experimentalists in astrophysical laboratories have focused their work on predicting the spectroscopic properties of cosmic dust particles more accurately. In contrast to the amorphous silicates in the diffuse interstellar medium, crystalline silicates are frequently observed in protoplanetary disks, which indicates their in-situ formation by thermal annealing or shocks (for a review see Ref. 16). An interesting result of astronomical observations of protoplanetary and circumstellar dust is the fact that the crystalline silicates are mostly magnesiumrich [17-19].

Extracting reliable dust properties from the thermal emission spectra of a mixture of dust particles requires precise knowledge of the optical properties for all possible constituents under various conditions. Crystalline silicates as the most important dust minerals, e.g., have very characteristic infrared spectra with many bands at wavelengths between $8 \mu \mathrm{m}$ and the submillimeter range. The details of band positions and band profiles, however, depend on the chemical composition, the grain morphology, and the grain temperature. This means that morphological 
information, e.g., can only been extracted from the spectra if the influence of the other parameters is sufficiently well understood and can be disentangled.

The grains condensed in circumstellar environments and modified by interstellar processes and even re-formed in the interstellar medium may be quite chaotic in composition and structure. Table 1 summarizes some of the chemical and structural properties which may characterize the particles on different length scales. The theoretical description of the optical properties of such particles can be very difficult. Even the "optical constants" (the complex refractive index $m=n+i k$ or the dielectric function $\epsilon=\epsilon_{1}+i \epsilon_{2}$ ) of the materials constituting the particle may be poorly known.

These facts require studies which have to be done in the laboratory, via synthesis and optical spectroscopy of materials representing important aspects of the cosmic solid matter [20, 21]. The investigations of these "cosmic dust analogs" do not necessarily aim at exactly reproducing cosmic dust. In our terminology dust analogs comprise also bulk matter or thin films prepared for the derivation of optical material constants. In addition, we also consider simple "model" particles (e.g., silica monospheres) used for the investigation of special aspects of particle optics (e.g., agglomeration effects).

Figure1 1 illustrates the various aspects of this approach. Two principal possibilities exist for the derivation of the optical properties of particles:

1. Calculation of cross sections from optical material data which may have been measured or taken from a data base. In most cases, the data will originate from a bulk or thin-film material since measurements on these geometrically simple systems are much easier and much more work has been done on them. Therefore one has the problem that the material may not be exactly the one one would like to have. Special material structures may only exist in the form of small grains (e.g., silicon nanoparticles, carbon onions). Additionally, theory has some problems with the calculation of spectra for complicated grain shapes in strong absorption bands. This will be discussed further in Sec. 4 .

2. Direct measurement of the optical properties on particle samples. This certainly is the preferred but also experimentally much more demanding possibility. One has to produce particles of the desired composition and structure and one has to store them in an environment as similar to the cosmic one as possible. This is sometimes problematic because conventional preparation of particulate samples for spectroscopy (i) uses matrices in which the particles are embedded and (ii) is not able to prevent agglomeration of the primary particles to larger clusters.

The next section will treat the basics of the first (computational) way, the light scattering theory, which also underlies the experimental way. Section 4 will deal with measurements of the material optical data from bulk and thin film samples as well as of extinction data of particulate samples.

\section{ASTRONOMICAL FEATURES AND DEVELOPMENTS IN LIGHT SCATTERING THEORY}

The optical properties of small particles can considerably deviate from those of bulk materials because of the occurrence of surface modes. The structure of the interface of small particles with the surrounding medium usually has strong effects on their optical properties. In addition, the shape and internal structure of the particles are important parameters in determining the position and shape of the observed astronomical features. Furthermore, the optical depth and the temperature structure of the dusty astronomical media are decisive for the occurrence of the solid-state bands and will determine if they are observed in emission, absorption, or selfabsorption. Protostars, deeply embedded in their parental molecular clouds, show absorption 
features due to molecular ices (e.g., $\mathrm{H}_{2} \mathrm{O}, \mathrm{CO}, \mathrm{CO}_{2}$ and $\mathrm{NH}_{3}$ ) and refractory silicates. In contrast, the spectra of protoplanetary disks around young stars are mostly characterized by a variety of silicate emission bands, produced in the optically thin disk atmosphere on top of the optically thick disk region.

For micron-sized grains classical electrodynamic theory can be applied using bulk optical constants. Textbooks which treat the classical electrodynamics of absorption and scattering of light by small particles are the publications by van de Hulst [22], Kerker [23], Bohren \& Huffman [24], Kokhanovsky [25], Mishchenko, Hovenier \& Travis [26], and Mishchenko, Travis \& Lacis [27].

The qualitative features of the absorption and scattering of light strongly depend on the ratio between the wavelength of the incident light $\lambda$ and the typical radius of the particle $a$. We can distinguish three regions:

1. $\lambda \ll a$ : This is the limit of geometrical optics, where the propagation of light is described by rays which are reflected and refracted at the surface of the scatterer according to Snell's law and the Fresnel formulae. For absorbing materials, light can penetrate only within the skin depth. Scattering, therefore, is mainly a surface effect and the extinction crosssection per volume goes roughly as $1 / a$.

2. $\lambda \sim a$ : This is the regime of wave optics, where both the angular and the wavelength dependence of the cross sections are dominated by interferences and resonances. For spherical particles, these can be calculated by the Lorenz-Mie theory [28].

3. $\lambda \gg a$ : This is the Rayleigh limit. If furthermore we have $\lambda \gg|m| a$, where $m$ is the (complex) refractive index of the particle, we are in the quasi-static limit. Then, both the incident and the internal field can be regarded as static fields. In this regime, phase shifts over the particle size are negligible. This implies that it is generally sufficient to consider only the dipolar electric mode.

The interaction of infrared radiation with sub-micron sized cosmic grains can generally be considered as a good example of the quasi-static case. However, particles with high imaginary parts of the refractive index (metals, semiconductors) may easily violate this condition even at infrared wavelengths. The same is true for laboratory measurements where it is not always guaranteed that the particles fulfill the condition for the quasi-static case.

For the sake of simplicity and physical insight, we will deal in the following only with the quasi-static case. The extinction cross section $C_{\text {ext }}$ is given by:

$$
C_{\text {ext }}=C_{\mathrm{abs}}+C_{\mathrm{sca}}
$$

where $C_{\text {abs }}$ and $C_{\text {sca }}$ are the absorption and scattering cross sections. According to Rayleigh's law $C_{\text {sca }} \propto k^{4}$ with the wavenumber $k=2 \pi / \lambda$. For $\lambda \gg a$ this means in practice that the extinction cross section equals the absorption cross section. The cross section for the radiation pressure can be computed via the formula

$$
C_{\text {pre }}=C_{\text {ext }}-C_{\text {sca }}\langle\cos \theta\rangle
$$

with $\langle\cos \theta\rangle$ the mean of the cosine of the scattering angle. Following the conventions in [24], the expression for the extinction cross section is given by:

$$
C_{\text {ext }}=k \operatorname{Im}(\alpha)
$$

with $\alpha$ being the polarizability. This quantity depends on the complex dielectric function, $\epsilon=$ $\epsilon_{1}+\mathrm{i} \epsilon_{2}$, of the particle material and on the dielectric function of the embedding medium, $\epsilon_{\mathrm{m}}$, 


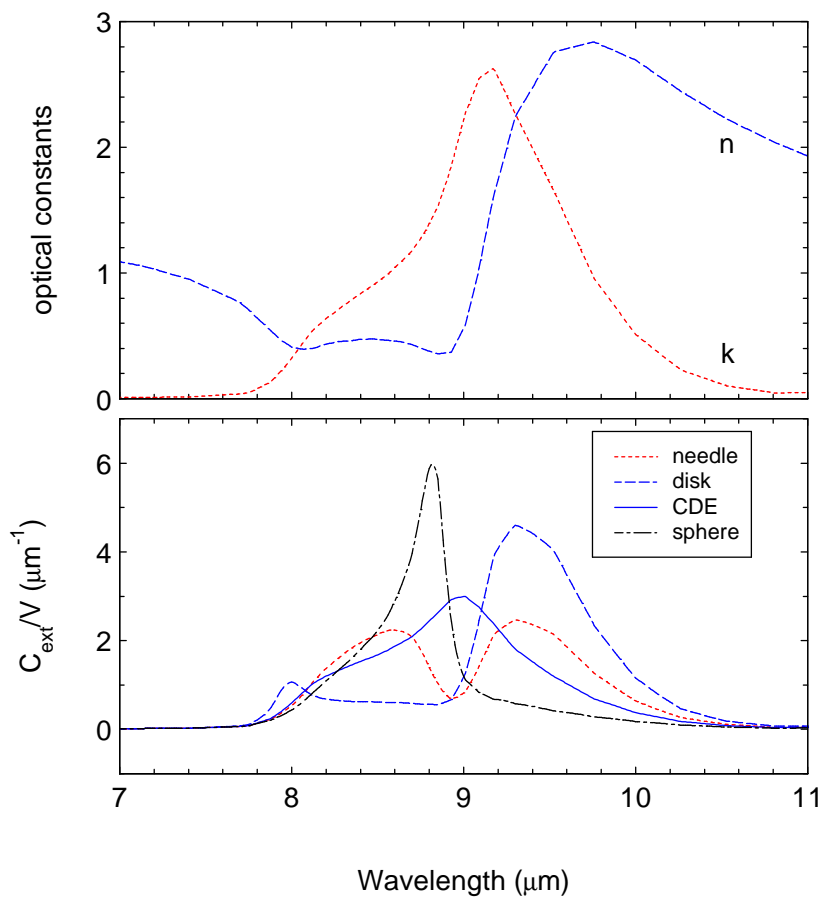

Fig. 2. Shape effects for $\mathrm{SiO}_{2}$ particles in the quasi-static limit. CDE refers to a continuous distribution of ellipsoidal shapes (Eq. 9).

which is in most cases a real wavelength-dependent number. In addition, the polarizability is determined by the shape of the particle.

For ellipsoids the polarizability, $\alpha_{\mathrm{i}}$, in an electric field parallel to one of its principal axes is given by

$$
\alpha_{\mathrm{i}}=V \frac{\epsilon-\epsilon_{\mathrm{m}}}{\epsilon_{m}+L_{\mathrm{i}}\left(\epsilon-\epsilon_{\mathrm{m}}\right)},
$$

where $L_{\mathrm{i}}$ are geometrical factors and $\mathrm{V}$ is the volume of the ellipsoid. The relation $L_{1}+L_{2}+L_{3}=1$ implies that only two of these factors are independent. In the case of prolate spheroids with equal minor axes of length $\mathrm{b}=\mathrm{c}$ and with eccentricity $e=\sqrt{1-c^{2} / a^{2}}$ (a the length of the major axis) the geometrical factors are given by

$$
L_{1}=\frac{1-e^{2}}{e}\left(-1+\frac{1}{2 e} \ln \frac{1+e}{1-e}\right)
$$

and $L_{2}=L_{3}=\left(1-L_{1}\right) / 2$. In the other case of oblate spheroids we have $a=b$ and $L_{1}=L_{2}=(1-$ $\left.L_{3}\right) / 2$ with

$$
L_{1}=\frac{\sqrt{e^{-2}-1}}{2 e^{2}}\left(\frac{\pi}{2}-\tan ^{-1}\left(\sqrt{e^{-2}-1}\right)\right)-\frac{e^{-2}-1}{2}
$$

The simplest case of light scattering in the quasi-static limit is that for a sphere where $a=b=c$ and $L_{\mathrm{i}}=1 / 3$. This gives $\alpha_{i}=\alpha$

$$
\alpha=3 V \frac{\epsilon-\epsilon_{\mathrm{m}}}{\epsilon+2 \epsilon_{\mathrm{m}}} .
$$

These equations demonstrate that resonances for particles surrounded by a non-aborbing medium occur close to wavelengths where the imaginary part of the dielectric function is close to zero and the real part fulfills the condition

$$
\epsilon_{1}=\epsilon_{m}\left(1-L_{\mathrm{i}}^{-1}\right)
$$


This immediately implies that the wavelengths of resonances depend on the shape of the particles. The resonances can only occur in regions where $\epsilon_{1}$ is negative (for a sphere $\epsilon_{1}=-2 \epsilon_{\mathrm{m}}$ ). Examples of astronomically relevant materials which have negative values of $\epsilon_{1}$ are $\mathrm{SiC}$ [29] and $\mathrm{SiO}_{2}[30]$ in the infrared and graphite in the UV [31]. For metal particles resonances even occur at wavelengths where the bulk material does not show any absorption band. In contrast, the lattice features of amorphous silicates do not show this behaviour.

In the case of lattice modes, the resonances are located between the transverse and logitudinal optical phonon frequencies. As an example, we show shape effects for $\mathrm{SiO}_{2}$ particles in Fig. 2 The equations also show that the positions of resonances depend on the surrounding medium - a fact which is often considered in astronomy in the case of dust models with core-mantle grains. For particles of arbitrary shape there are no analytical solutions of the light scattering problem (even in the quasi-static limit) available. Numerical models frequently used for non-spherical particles are the separation of variables method, the T-matrix method and the discrete dipole (or multipole) approximation (see, e.g., Ref. 32). The treatment of anisotropic materials gets more complicated, but is still possible [33].

In reality, we always expect a distribution of particle shapes. The difficulty both in astronomical applications and in laboratory experiments is to evaluate this shape distribution. A simple model for a distribution of particle shapes is the continuous distribution of ellipsoids (CDE), which by averaging Eq. (4) over all $\mathrm{L}_{i}$ leads to

$$
\alpha_{a v}=V \frac{2 \epsilon}{\epsilon-1} \log \epsilon,
$$

where $\log \epsilon$ denotes the principal value of the logarithm of the complex number $\epsilon$ (see Bohren \& Huffman 1983). The CDE assumes equal probability for the presence of every shape and averages over all orientations. This model can be used to estimate in an approximate way how important shape effects for a special material could be.

Recently, Min et al. [34] have shown that an arbitrary grain shape can be described by a distribution of geometrical factors $\mathrm{P}(\mathrm{L})$, which can be derived from a spatial discretization of the particle shape. Such distributions containing the precise shape information can be averaged for representative shapes of an ensemble of particles and can be used to calculate the ensemble polarizability

$$
\alpha_{a v}=V \int_{0}^{1} P(L) \alpha(L) d L
$$

with $\alpha(\mathrm{L})$ according to Eq. (4). This "distribution of form factors (DFF)" model has been proven to be able to successfully describe experimental IR spectra for different grain shapes of submicron-sized particles and to predict the influence of an embedding medium correctly [35]. $\mathrm{P}(\mathrm{L})$ distributions - DFFs - can be derived by fitting experimental spectra and typical DFFs for irregular and roundish grains have been extracted (see also Sec. 4.3.). Compared to other models, the use of these DFFs allows a fast computation of quite realistic band profiles for real particulates.

Here we should stress that all of the previous considerations are only valid in the quasi-static case and for isolated particles. Another shortcoming of this and all similar models is that for crystallographic anisotropic materials, the polarizability has to be calculated with the different sets of optical constants separately. This neglects possible interaction of dipoles with different orientations and can lead to errors in some cases [35].

In dense regions of interstellar matter (dense molecular cloud cores, circumstellar disks and envelopes), the submicron-sized grains can coagulate and finally form larger particles [36, 37]. For a description of the interaction of electromagnetic radiation with fluffy aggregates composed of individual particles two distinct approaches are possible: 
1. "Deterministic" approach: The frequency-domain Maxwell equation is solved for an individual cluster. The resulting cross sections are calculated for many clusters and averaged over the ensemble and the orientation of the clusters. An advantage of this approach is that for special systems (e.g., clusters of spheres), exact solutions of the problem do exist. For computational reasons, however, these methods are limited to either comparatively small clusters or moderately absorbing systems. For larger clusters of highly absorbing matter (as in the case of graphitic carbonaceous grains and metal clusters), one still has to rely on approximations. Examples of this kind of approach are the discrete dipole and multipole approximations (DDA/DMA) and the extended Mie theory for multisphere aggregates.

2. "Statistical" approach: The equations are formulated in terms of statistically relevant quantities only (e.g., average radial density function of the clusters, density correlation function) without any explicit treatment of the properties of individual particles. Whereas a given cluster generally does not have any symmetry, statistical averages show rotational invariance, unless alignment mechanisms (magnetic fields, winds) break this symmetry. The advantage of this approach is that only the necessary information (ensemble and orientation averaged quantities) enter the calculations. Examples of this approach are the different effective medium theories, the strong permittivity fluctuation theory, and the "Distribution of Hollow Spheres" model [38]. The above-mentioned DFF model belongs also to this class.

Benchmark results for these methods and astronomically relevant materials have been published by Stognienko et al. [39], Michel et al. [40], Xu \& Gustafson [41], and Voshchinnikov et al. [32]. The interested reader should consult these papers for more detailed information. The properties of porous and fractal cosmic grains have recently found renewed interest and were calculated by various authors [42, 45].

The $10 \mu \mathrm{m}$ silicate feature has been frequently used to trace the particle size in protoplanetary disks. Infrared spectroscopy of disks have shown the presence of flat-top profiles, indicative of the presence of micron-sized particles which are larger than the typical particle sizes in the diffuse interstellar medium (e.g., Refs. 46 and 47). The influence of porosity and aggregate structure on this important astronomical dust feature has been investigated by Min et al. [48] and Voshchinnikov \& Henning [49].

\section{DEVELOPMENTS IN SPECTROSCOPIC EXPERIMENTS}

\subsection{Spectroscopic data of dust minerals}

One type of information which may be possible to extract from infrared emission spectra of cosmic dusty media is the chemical composition and mineralogical structure of the dust minerals at the present stage of evolution of the respective object. In a great effort to provide experimental dust spectra for comparison with the astronomical infrared data, the spectroscopic properties of the (likely) major dust minerals have been investigated in different laboratories and have been made available to the astronomical community. For example, the infrared spectra of crystalline silicates with different iron to magnesium ratios have been studied in great detail in the last decade, especially by the group of C. Koike at Kyoto Pharmaceutical University (e.g., Refs. 50 and 51) by manufacturing and measuring synthetic crystals of variable composition.

The technique applied for the infrared spectroscopy is usually transmission measurements of powders embedded into solid matrices (pellets made of $\mathrm{KBr}$, CsI or polyethylene depending on the wavelength range of the spectroscopic measurement). In order to reach the Rayleigh case (size smaller than wavelength), the particles are mechanically ground and size-separated by sedimentation. The fine-grained particles are dispersed among the matrix material as homogeneously as possible at a certain mass ratio. The pellets then contain the particles at a known 


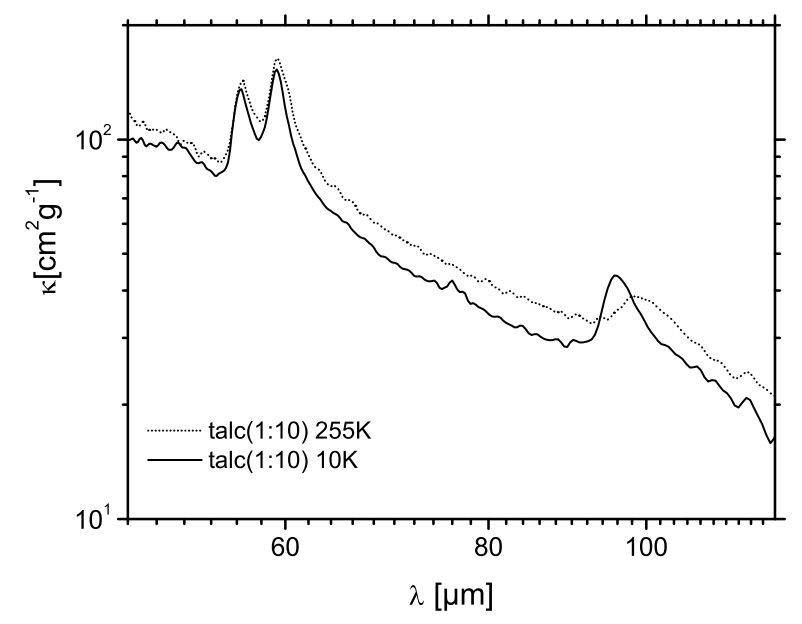

Fig. 3. Mass absorption coefficient of talc $\left(\mathrm{Mg}_{3}\left[\mathrm{Si}_{4} \mathrm{O}_{10} \mid(\mathrm{OH})_{2}\right]\right)$ at far-infrared wavelengths for two different temperatures. The ratio 1:10 (by mass) refers to the dilution of the talc powder by polyethylene in the pellet - after Ref. 55.

column density $\sigma=m / A$, with A being the pellet cross sectional area. The advantages of this technique are easy preparation, small sample consumption and quantitative determination of mass absorption coefficients $\kappa=-\ln (T) / \sigma$ from the spectral transmission of the pellet. Due to Kirchhoffs law, absorption coefficients can be directly used as emissivity of dust grains in thermal emission spectra.

The same technique has been employed to study the variations of band positions and band profiles with temperature, especially for low temperatures down to a few Kelvin. Because of the anharmonicity of the vibrational potentials of the lattice modes causing the infrared bands, the bands tend to get narrower and to shift to smaller wavelengths as the vibrational levels are de-populated with lowering T. Such studies have been performed e.g., by the groups in London, Kyoto, St. Louis and Jena for olivine particles [52-54] and for phyllosilicates (see Fig. 36 [55-57]. It has been found that the sharpness and wavelength position of certain silicate far-infrared bands might be actually good indicators of the dust temperature.

The drawback of the powder transmission spectroscopy in pellets, generally, is the possible influence of the polarisation of the embedding medium on the spectra. It can be easily shown for instance by Mie calculations that such an influence exists (see Sec. 3). In the general case of nonspherical or even irregular particle shapes, however, it is difficult to correct for this influence, although it is possible if the material's optical constants are known (see also below) [58, 59].

\subsection{Optical constants}

Most astrophysicists modeling thermal dust emission spectra rely on calculated rather than experimentally measured spectra, because of the flexibility of such calculations if parameters such as grain sizes have to be varied. The material properties enter into these calculations usually in the form of the so-called "optical constants". This term denotes the response function which characterizes the reaction of a material to an external (time-variable) electromagnetic field: the complex refractive index $m=n+i k$. If magnetic material properties can be neglected, $m$ is the square root of the (also complex) dielectric function $\varepsilon$ which alternatively can be used as optical constants.

It has to be noted that these quantities are macroscopic in character, therefore they loose their meaning for small clusters and molecules. In the transition region from solids to molecules the 


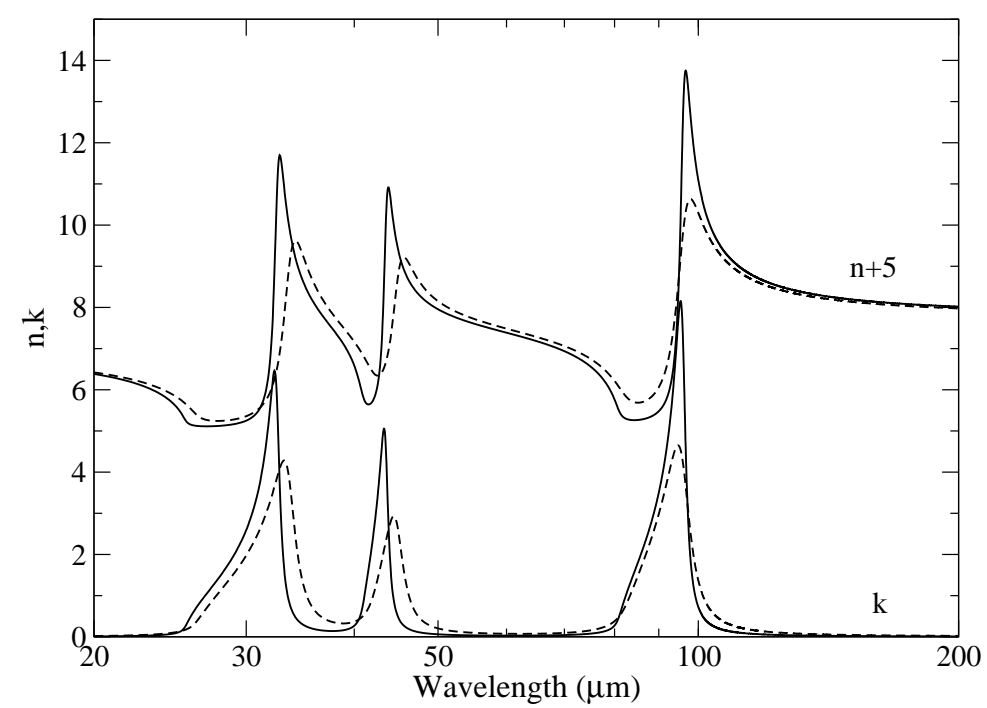

Fig. 4. Far-infrared optical constants of calcite at temperatures of $10 \mathrm{~K}$ (solid lines) and $300 \mathrm{~K}$ (dashed lines) - after Ref. 61.

introduction of size-dependent optical material properties may be a reasonable way to include e.g., the confinement of charge carriers to the limited particle volume [60].

The term optical constants is a bit misleading since they are strongly frequency-dependent. The dispersion with frequency is determined by resonances of the electronic system, of the ionic lattice and, at very low frequencies, by relaxation of permanent dipoles. In these frequency regions absorption becomes strong (high $k$ ) and $n$ shows "anomalous dispersion", i.e. decreases with frequency. In many cases, this behaviour can be described by Lorentzian oscillators.

Frequency-dependent optical "constants" of solid materials can be found in a number of databases which are available either in the form of books or electronic media. The most important database in book-form is the "Handbook of Optical Constants of Solids" edited by E.D. Palik that currently consists of three volumes which appeared in 1985, 1991 and 1998 [62 64]. These books are recommended since they comprise detailed discussions of the origin and errors of each dataset which may prevent the user from uncritical application of these data.

The Jena-St.Petersburg-Heidelberg database of optical constants [65] which is especially dedicated to cosmic dust applications can be found via the web pages http://www.astro.uni-jena.de and http://www.mpia-hd.mpg.de/HJPDOC.

As mentioned already, nearly all of these optical constants have been measured either on bulk samples or on thin films. The reason for that is that the planar geometry of films or coatings is much easier to describe in deriving optical constants from a measured spectrum than are irregularly shaped particles. However, even in the case of ideal bulk measurements, the determination of optical constants over a wide frequency or wavelength range is not a simple task. Since the material absorption in different spectral regions usually differs by many orders of magnitude, for the determination of $k$ either transmission measurements on samples of very different thicknesses (from centimeter down to submicron scales) or transmission and reflection measurements have to be combined [66]. Many crystals show an anisotropy in their optical constants. In these cases, measurements with polarized light along the different axes of the crystal have to be carried out which require careful orientation of the crystal and alignment of the polarizers.

In regions of strong absorption, as mainly considered here, usually reflection measurements have to be used and either the Kramers-Kronig relations (see Ref. 24) or a parametrization of the optical constants as a sum of Lorentzian oscillators have to be applied in order to obtain both 


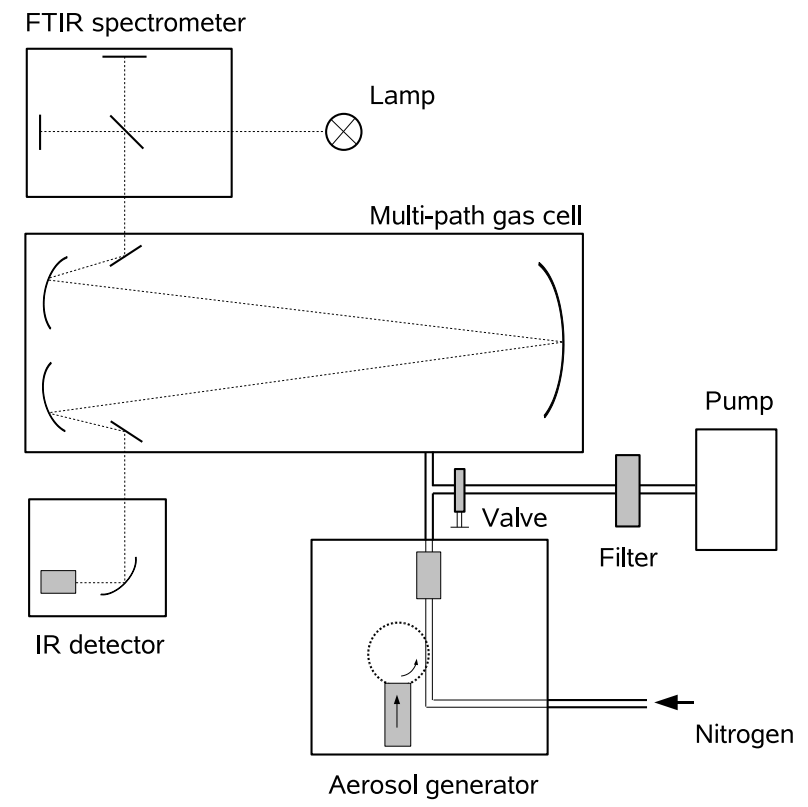

Fig. 5. Schematic structure of the aerosol spectroscopy setup.

the real and imaginary parts of the optical constants. As already mentioned above, the optical constants of cosmic dust analogs may strongly depend on the temperature of the material, so that temperature-dependent data are desired.

Despite of the difficulties inherent to the preparation of samples and the measurement and data evaluation procedures in obtaining such data, some progress has also been made in the measurements of the optical constants for dust materials within the last years. For instance, optical constants of amorphous magnesium silicates with a variable magnesium content have been measured in a wide wavelength range [67]. For crystalline silicates, the optical constants of minerals of the olivine series (composition $(\mathrm{Mg}, \mathrm{Fe})_{2} \mathrm{SiO}_{4}$ ) have been re-measured for different magnesium-to-iron ratios (e.g., Refs. 68 and 69) and even at temperatures down to $50 \mathrm{~K}$ (for $\mathrm{Mg}_{2} \mathrm{SiO}_{4}$, [70]). More temperature-dependent optical constants have been derived for carbonates [61], including the far-infrared wavelength range (see Fig. 4).

\subsection{Aerosol measurements and grain morphology}

The influence of the grain morphology and of the embedding into matrices has always been a concern in the use of experimental spectra for comparison with observed ones. As demonstrated in Sec. 3, the absorption band profiles of solid particles depend on the grain shape and on the electromagnetic polarisability (the refractive index) of the ambient medium.

When comparing spectra of crystalline silicates measured in different laboratories, it has been realized that band profiles and peak positions showed systematic differences, which were supposedly related to the morphological properties of the particulates such as the grain shape (influenced by the powder grinding technology) and perhaps the (unknown) state of agglomeration inside the pellets [54,71]. On the other hand, spectra calculated from the material's optical constants assuming a spherical grain shape failed completely to reproduce both observed and measured spectra. Simple statistical approaches to more realistic grain shape models such as the Continuous Distribution of Ellipsoids (see Sec. 3) gave much better, but still not sufficient, results [68]. 

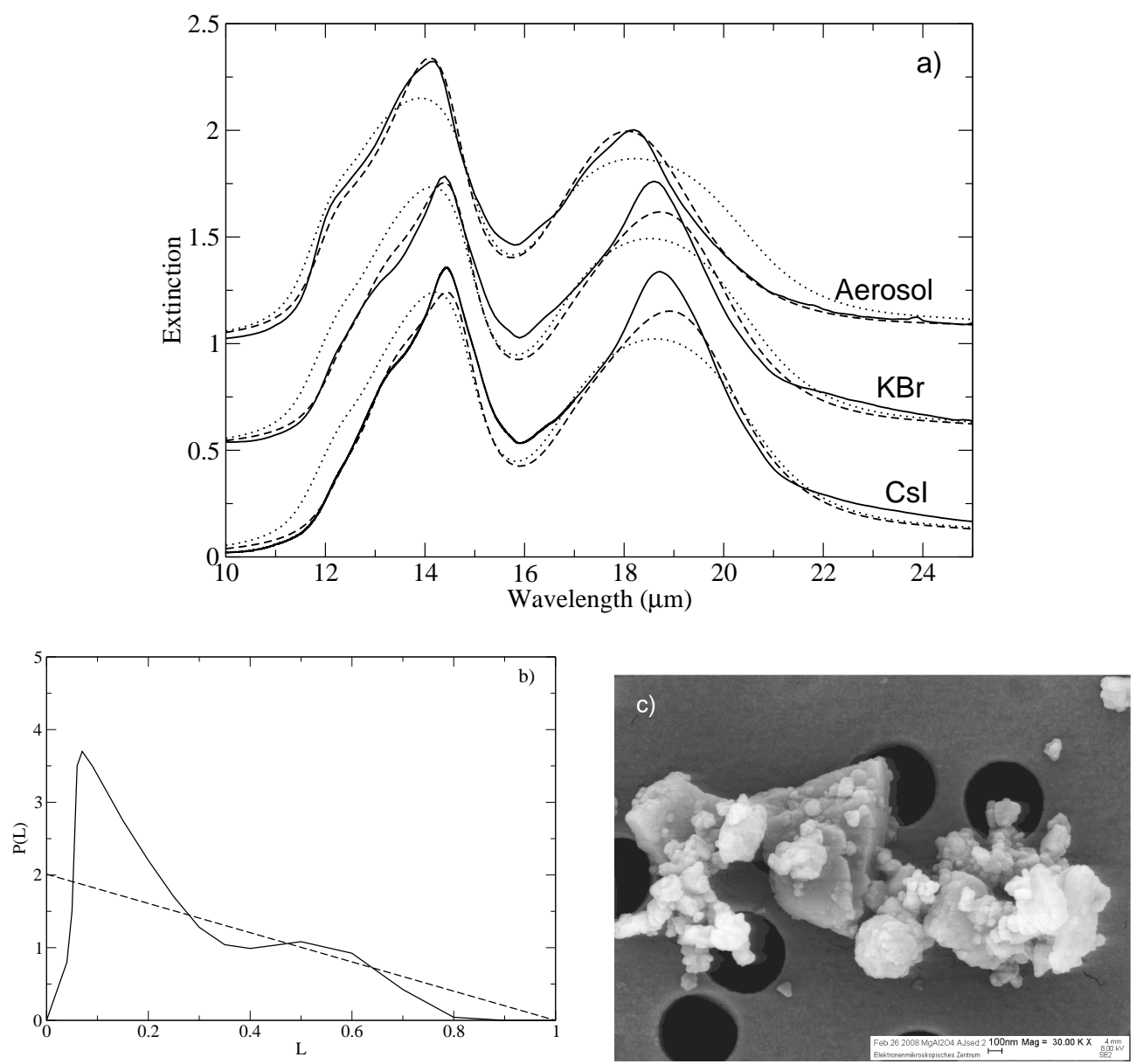

Fig. 6. (a) Absorption spectra of irregular-shaped spinel particles measured in different media (nitrogen gas, $\mathrm{KBr}$ and CSi pellets - solid lines) compared to simulated spectra using the CDE (dotted line) and DFF (dashed line) models. (b) The shape factor distribution used for the DFF calculations (solid line) compared to that of the $\operatorname{CDE}(\mathrm{P}(\mathrm{L})=2(1-\mathrm{L})$, dashed line). (c) Electron micrograph of the particles. The black filter holes have a diameter of $0.5 \mu \mathrm{m}$. 
From the experimental side, an important step forward was made a few years ago, when the aerosol spectroscopy technique was introduced into the measurement of experimental comparison spectra for astronomical puposes [72]. In contrast to the pellet method, this technique proved to be suitable not only for avoiding any influence of an embedding medium onto the spectra, but also for a detailed study of morphological effects on infrared spectra of dust samples [73]. The reason for this is that a direct electron microscopic investigation of the aerosol particles is routinely possible after the spectroscopic measurement.

The experimental setup for aerosol infrared spectroscopy as used at the astrophysical laboratory of the AIU Jena consists of a White-type multi-path gas cell connected to a Bruker 113v FTIR spectrometer and equipped with an "aerosol generator" for dispersing fine powders in nitrogen gas and filling the gas cell with this aerosol (see Fig. (5). The aerosol generator makes use of a Palas RBG 1000 rotating-brush particle disperser and is also equipped with an impactortype grain-size discriminator in order to allow only small particles to reach the gas cell. The aerosol formed of these submicron-sized particles in a gas at about normal pressure is stable for several minutes, sufficient for FTIR measurements. After the measurement, a part of the particles are filtered from the aerosol for scanning electron microscopic (SEM) inspection. The drawbacks of this technique are that the column density of the particles is not precisely known, making a quantitative measurement of the absorption coefficient difficult, the large sample consumption, and the limitation to a certain particle size determined by the sedimentation speed.

By comparing the aerosol-measured infrared spectra with pellet spectra, the influence of the embedding medium on the band profiles has been made clear for a large number of oxide [74] and silicate [75] mineral "dust samples". The internet database making such aerosol spectra publically available (http://elbe.astro.uni-jena.de) contains also spectra of particles embedded in cesium iodide (CsI) pellets for comparison. The latter are usually characterized by infrared bands peaking at longer wavelengths. This does not mean that the bands are just shifted, but that surface modes closer to the transverse optical lattice resonance get a higher weight in the band profiles (see below).

In the course of these aerosol measurements, great attention has been given to morphological effects in the spectra and it has been confirmed that grain shapes play a big role for the band profiles. For several compounds it has been possible to measure powders with different grain shapes, for instance more roundish ones vs. more irregular, sharp-edged shapes. The respective infrared bands were found to be characteristically different, for the roundish grains often revealing a short-wavelength peak and a long-wavelength shoulder, whereas irregular grains produced long- or intermediate wavelength single peaks [35,74].

As mentioned in Sec. 3, the DFF model can successfully be used to reproduce these band profiles. Figure 6 demonstrates this for irregularly shaped spinel $\left(\mathrm{MgAl}_{2} \mathrm{O}_{4}\right)$ particles measured in various environments (aerosol, pellets). The form factor distribution used in these computations is also shown. It has to be noted again that this model, as many others, is only exact in the case of particles composed of an isotropic material.

\section{CONCLUSIONS}

The measurements and the theoretical calculations of optical properties of cosmic grains play an important role in a wide range of astrophysical applications. The collection of relevant data for well-characterized materials over a broad wavelength range has made great progress over the last two decades. Here we want to stress that the characterization of the physical and chemical structure of the materials is an important part of the study of cosmic dust analogs. An interesting direction of future research will be the comparison of the properties of cosmic dust grains collected by interplanetary space missions such as the Stardust mission (e.g., Ref. 76) with synthetic materials produced in the laboratory.

The combination of condensation experiments carried out under conditions similar to cosmic environments and advanced spectroscopic methods is certainly the future of solid-state 
astrophysics. Mass spectroscopy has to be used to characterize the transition from atoms and molecules to small clusters and solids. In addition, the change of material properties under the bombardment of cosmic rays and irradiation with intense X-ray and UV photons has to be better understood. Another important factor can be thermal annealing cycles of the grains.

The application of laboratory data for the interpretation of astronomically measured fluxes relies on the knowledge of the density and temperature structure of the considered dusty regions. Radiative transfer calculations have to be carried out for deeply embedded objects such as protostars and active galactic nuclei. In the case of very small particles the effects of quantum heating have to be taken into account. Even for the interpretation of spectra of optically thin configurations, the dust temperature and density distributions have to be known.

\section{Acknowledgments}

We gratefully acknowledge the collaboration with our colleagues Dr. Akemi Tamanai, Gabriele Born, and Walter Teuschel (all AIU Jena) on the laboratory experiments and Dr. Michiel Min (Astronomical Institute Utrecht) on the simulations of dust spectra. Our research has been supported by the Deutsche Forschungsgemeinschaft with grants MU 1164/5,6.

\section{References}

[1] L. J. Allamandola and A. G. G. M. Tielens, Eds., Interstellar Dust: Proceedings of the 135th Symposium of the International Astronomical Union, IAU Symposium 135, Kluwer, Dordrecht (1989).

[2] J. S. Mathis, "Dust models with tight abundance constraints," Astrophys. J. 472, 643-655 (1996) [doi: 10.1086/178094].

[3] A. Li and J. M. Greenberg, "A unified model of interstellar dust," Astron. Astrophys. 323, 566-584 (1997).

[4] V. Zubko, E. Dwek, and R. G. Arendt, "Interstellar dust models consistent with extinction, emission, and abundance constraints," Astrophys. J. Suppl. 152, 211-249 (2004) [doi: $10.1086 / 382351]$.

[5] B. T. Draine and A. Li, "Infrared emission from interstellar dust. IV. The silicategraphite-PAH model in the post-Spitzer era," Astrophys. J. 657, 810-837 (2007) [doi: 10.1086/511055].

[6] A. N. Witt, G. C. Clayton, and B. T. Draine, Eds., Astrophysics of Dust, Astron. Soc. Pacific Conf. Ser. 309 (2004).

[7] Th. Henning, E. Grün, and J. Steinacker (eds.), Cosmic Dust - Near and Far, Astron. Soc. Pacific Conf. Ser., in press (2009).

[8] J. Dorschner and T. Henning, "Dust metamorphosis in the galaxy," Astron. Astrophys. Rev. 6, 271-333 (1995) [doi: 10.1007/BF00873686].

[9] E. F. van Dishoeck, "ISO spectroscopy of gas and dust: From molecular clouds to protoplanetary disks," Annu. Rev. Astron. Astrophys. 42, 119-167 (2004) [doi: 10.1146/annurev.astro.42.053102.134010].

[10] W. A. Schutte, "Ices in the interstellar medium," in Laboratory Astrophysics and Space Research, P. Ehrenfreund, C. Krafft, H. Kochan, and V. Pirronello, Eds., Astrophysics and Space Science Library 236, 69, Kluwer, Dordrecht (1998).

[11] W. A. Schutte, "Ice evolution in the interstellar medium," in Solid Interstellar Matter: The ISO Revolution, L. D'Hendecourt, C. Joblin, and A. Jones, Eds., 183, EDP and Springer, Berlin (1999).

[12] T. Henning and F. Salama, "Carbon in the universe," Science 282, 2204-2210 (1998) [doi: 10.1126/science.282.5397.2204]. 
[13] F. Salama, "Polycyclic aromatic hydrocarbons in the interstellar medium: A review," in Solid Interstellar Matter: The ISO Revolution, L. D'Hendecourt, C. Joblin, and A. Jones, Eds., 65, EDP and Springer, Berlin (1999).

[14] C. Jäger, F. J. Molster, J. Dorschner, T. Henning, H. Mutschke, and L. B. F. M. Waters, "Steps toward interstellar silicate mineralogy. IV. The crystalline revolution," Astron. Astrophys. 339, 904-916 (1998).

[15] L. B. F. M. Waters, F. J. Molster, and F. Kemper, "The Mineralogy of Interstellar and Circumstellar Dust in Galaxies," in Astromineralogy, 2nd rev. ed., Th. Henning, Ed., Springer, Berlin, in press (2010).

[16] Th. Henning and G. Meeus, "Dust processing and mineralogy in protoplanetary accretion disks," in Physical Processes in Circumstellar Disks around Young Stars, P.J.V. Garcia, Ed., Theoretical Astrophysics Series, Chicago University Press, in press (2010).

[17] J. Bouwman, G. Meeus, A. de Koter, S. Hony, C. Dominik, and L. B. F. M. Waters, "Processing of silicate dust grains in Herbig Ae/Be systems," Astron. Astrophys. 375, 950 962 (2001) [doi: 10.1051/0004-6361:20010878].

[18] J. Bouwman, T. Henning, L. A. Hillenbrand, M. R. Meyer, I. Pascucci, J. Carpenter, D. Hines, J. S. Kim, M. D. Silverstone, D. Hollenbach, and S. Wolf, "The formation and evolution of planetary systems: Grain growth and chemical processing of dust in T Tauri systems," Astrophys. J. 683, 479-498 (2008) [doi: 10.1086/587793].

[19] A. Juhász, J. Bouwman, Th. Henning, B. Acke, M.E. van den Ancker, G. Meeus, et al., "Dust evolution in protoplanetary disks around Herbig Ae/Be stars - The Spitzer view," Astrophys. J., in press (2010).

[20] L. Colangeli, T. Henning, J. R. Brucato, D. Clément, D. Fabian, O. Guillois, F. Huisken, C. Jäger, E. K. Jessberger, A. Jones, G. Ledoux, G. Manicó, V. Mennella, F. J. Molster, H. Mutschke, V. Pirronello, C. Reynaud, J. Roser, G. Vidali, and L. B. F. M. Waters, "The role of laboratory experiments in the characterisation of silicon-based cosmic material," Astron. Astrophys. Rev. 11, 97-152 (2003) [doi: 10.1007/s00159-002-0017-x].

[21] C. Jäger, F. Huisken, H. Mutschke, and Th. Henning, "Analogs of Cosmic Dust" in Cosmic Dust - Near and Far, Th. Henning, E. Grün, and J. Steinacker, Eds., Astron. Soc. Pacific Conf. Ser., in press (2009).

[22] H. C. van de Hulst, Light Scattering by Small Particles, Wiley, New York (1957).

[23] M. Kerker, The Scattering of Light and other Electromagnetic Radiation., Academic Press, New York (1969).

[24] C. F. Bohren and D. R. Huffman, Absorption and Scattering of Light by Small Particles, Wiley, New York (1983).

[25] A. A. Kokhanovsky, Light Scattering Media Optics: Problems and Solutions, Praxis Publishing, Chichester (2004).

[26] M. I. Mishchenko, J. W. Hovenier, and L. D. Travis, Light Scattering by Nonspherical Particles : Theory, Measurements, and Applications, Academic Press, San Diego (2000).

[27] M. I. Mishchenko, L. D. Travis, and A. A. Lacis, Scattering, Absorption, and Emission of Light by Small Particles, Cambridge University Press, Cambridge (2002).

[28] G. Mie, "Beiträge zur Optik trüber Medien, speziell kolloidaler Metallösungen," Ann. Phys. Lpz. 330, 377-445 (1908) [doi: 10.1002/andp.19083300302].

[29] H. Mutschke, A. C. Andersen, D. Clément, T. Henning, and G. Peiter, "Infrared properties of SiC particles," Astron. Astrophys. 345, 187-202 (1999).

[30] T. Henning and H. Mutschke, "Low-temperature infrared properties of cosmic dust analogues," Astron. Astrophys. 327, 743-754 (1997).

[31] E. A. Taft and H. R. Philipp, "Optical properties of graphite," Phys. Rev. 138, 197-202 (1965) [doi: 10.1103/PhysRev.138.A197]. 
[32] N. V. Voshchinnikov, V. B. Il'in, T. Henning, B. Michel, and V. G. Farafonov, "Extinction and polarization of radiation by absorbing spheroids: shape/size effects and benchmark results.," J. Quant. Spectrosc. Radiat. Transf. 65, 877-893 (2000) [doi: 10.1016/S00224073(99)00159-4].

[33] B. Michel, Th. Henning, C. Jäger, and U. Kreibig, "Optical extinction by spherical carbonaceous particles" Carbon 37, 391-400 (1999) [doi: 10.1016/S0008-6223(98)002012].

[34] M. Min, J. W. Hovenier, C. Dominik, A. de Koter, and M. A. Yurkin, "Absorption and scattering properties of arbitrarily shaped particles in the Rayleigh domain," J. Quant. Spectrosc. Radiat. Transf. 97, 161-180 (2006) [doi: 10.1016/j.jqsrt.2005.05.059].

[35] H. Mutschke, M. Min, and A. Tamanai, "Laboratory-based grain-shape models for simulating dust infrared spectra," Astron. Astrophys. 504, 875-882 (2009) [doi: 10.1051/00046361/200912267].

[36] S. V. W. Beckwith, T. Henning, and Y. Nakagawa, "Dust properties and assembly of large particles in protoplanetary disks," in Protostars and Planets IV, V. Mannings, A.P. Boss, and S.S. Russell, Eds., Univ. Arizona Press, Tucson, 533-558 (2000).

[37] A. Natta, L. Testi, N. Calvet, T. Henning, R. Waters, and D. Wilner, "Dust in protoplanetary disks: Properties and evolution," in Protostars and Planets V, B. Reipurth, D. Jewitt, and K. Keil, Eds., Univ. Arizona Press, Tucson, 767-781 (2007).

[38] M. Min, J. W. Hovenier, and A. de Koter, "Modeling optical properties of cosmic dust grains using a distribution of hollow spheres," Astron. Astrophys. 432, 909-920 (2005) [doi: 10.1051/0004-6361:20041920].

[39] R. Stognienko, T. Henning, and V. Ossenkopf, "Optical properties of coagulated particles.," Astron. Astrophys. 296, 797-809 (1995).

[40] B. Michel, T. Henning, R. Stognienko, and F. Rouleau, "Extinction properties of dust grains: A new computational technique," Astrophys. J. 468, 834-841 (1996) [doi: 10.1086/177739].

[41] Y. Xu and B. Å. S. Gustafson, "Comparison between multisphere light-scattering calculations: Rigorous solution and discrete-dipole approximation," Astrophys. J. 513, 894-909 (1999) [doi: 10.1086/306892].

[42] N. V. Voshchinnikov, V. B. Il'in, and T. Henning, "Modelling the optical properties of composite and porous interstellar grains," Astron. Astrophys. 429, 371-381 (2005) [doi: 10.1051/0004-6361:200400081].

[43] Y. Shen, B. T. Draine, and E. T. Johnson, "Modeling porous dust grains with ballistic aggregates. I. Geometry and optical properties," Astrophys. J. 689, 260-275 (2008) [doi: 10.1086/592765].

[44] Y. Shen, B. T. Draine, and E. T. Johnson, "Modeling porous dust grains with ballistic aggregates. II. Light scattering properties," Astrophys. J. 696, 2126-2137 (2009) [doi: 10.1088/0004-637X/696/2/2126].

[45] M. Min, J. W. Hovenier, L. B. F. M. Waters, and A. de Koter, "The infrared emission spectra of compositionally inhomogeneous aggregates composed of irregularly shaped constituents," Astron. Astrophys. 489, 135-141 (2008) [doi: 10.1051/0004-6361:200809534].

[46] R. van Boekel, M. Min, L. B. F. M. Waters, A. de Koter, C. Dominik, M. E. van den Ancker, and J. Bouwman, "A $10 \mu \mathrm{m}$ spectroscopic survey of Herbig Ae star disks: Grain growth and crystallization," Astron. Astrophys. 437, 189-208 (2005) [doi: 10.1051/00046361:20042339].

[47] A. Sicilia-Aguilar, L. W. Hartmann, D. Watson, C. Bohac, T. Henning, and J. Bouwman, "Silicate dust in evolved protoplanetary disks: Growth, sedimentation, and accretion," Astrophys. J. 659, 1637-1660 (2007) [doi: 10.1086/512121]. 
[48] M. Min, C. Dominik, J. W. Hovenier, A. de Koter, and L. B. F. M. Waters, "The 10 $\mu \mathrm{m}$ amorphous silicate feature of fractal aggregates and compact particles with complex shapes," Astron. Astrophys. 445, 1005-1014 (2006) [doi: 10.1051/0004-6361:20053212].

[49] N. V. Voshchinnikov and T. Henning, "Is the silicate emission feature only influenced by grain size?," Astron. Astrophys. 483, L9-L12 (2008) [doi: 10.1051/00046361:200809697].

[50] H. Chihara, C. Koike, A. Tsuchiyama, S. Tachibana, and D. Sakamoto, "Compositional dependence of infrared absorption spectra of crystalline silicates. I. Mg-Fe pyroxenes," Astron. Astrophys. 391, 267-273 (2002) [doi: 10.1051/0004-6361:20020791].

[51] C. Koike, H. Chihara, A. Tsuchiyama, H. Suto, H. Sogawa, and H. Okuda, "Compositional dependence of infrared absorption spectra of crystalline silicate. II. Natural and synthetic olivines," Astron. Astrophys. 399, 1101-1107 (2003) [doi: 10.1051/00046361:20021831].

[52] J. E. Bowey, C. Lee, C. Tucker, A. M. Hofmeister, P. A. R. Ade, and M. J. Barlow, "Temperature effects on the 15-85 $\mu \mathrm{m}$ spectra of olivines and pyroxenes," Mon. Not. R. Astron. Soc. 325, 886-896 (2001) [doi: 10.1046/j.1365-8711.2001.04523.x].

[53] H. Chihara, C. Koike, and A. Tsuchiyama, "Low-temperature optical properties of silicate particles in the far-infrared region," Proc. Astron. Soc. Jpn. 53, 243-250 (2001).

[54] C. Koike, H. Mutschke, H. Suto, T. Naoi, H. Chihara, T. Henning, C. Jäger, A. Tsuchiyama, J. Dorschner, and H. Okuda, "Temperature effects on the mid-and far-infrared spectra of olivine particles," Astron. Astrophys. 449, 583-596 (2006) [doi: 10.1051/0004-6361:20053256].

[55] C. Koike and H. Shibai, "Optical constants of hydrous silicates from 7 microns to 400 microns," Mon. Not. R. Astron. Soc. 246, 332-336 (1990).

[56] A. M. Hofmeister and J. E. Bowey, "Quantitative infrared spectra of hydrosilicates and related minerals," Mon. Not. R. Astron. Soc. 367, 577-591 (2006) [doi: 10.1111/j.13652966.2006.09894.x].

[57] H. Mutschke, S. Zeidler, T. Posch, F. Kerschbaum, A. Baier, and T. Henning, "Farinfrared spectra of hydrous silicates at low temperatures. Providing laboratory data for Herschel and ALMA," Astron. Astrophys. 492, 117-125 (2008) [doi: 10.1051/00046361:200810312].

[58] R. Papoular, M. Cauchetier, S. Begin, and G. Lecaer, "Silicon carbide and the $11.3 \mu \mathrm{m}$ feature," Astron. Astrophys. 329, 1035-1044 (1998).

[59] D. Clément, H. Mutschke, R. Klein, and T. Henning, "New laboratory spectra of isolated $\beta$-SiC nanoparticles: Comparison with spectra taken by the Infrared Space Observatory," Astrophys. J. 594, 642-650 (2003) [doi: 10.1086/376864].

[60] U. Kreibig, and M. Vollmer, Optical Properties of Metal Clusters, Springer, Berlin (1995).

[61] T. Posch, A. Baier, H. Mutschke, and T. Henning, "Carbonates in space: The challenge of low-temperature data," Astrophys. J. 668, 993-1000 (2007) [doi: 10.1086/521390].

[62] E. D. Palik, Handbook of Optical Constants of Solids, Academic Press, Orlando (1985).

[63] E. D. Palik, Handbook of Optical Constants of Solids II, Academic Press, San Diego (1991).

[64] E. D. Palik, Handbook of Optical Constants of Solids III, Academic Press, San Diego (1998).

[65] T. Henning, V. B. Il'in, N. A. Krivova, B. Michel, and N. V. Voshchinnikov, "WWW database of optical constants for astronomy," Astron. Astrophys. Suppl. 136, 405-406 (1999) [doi: 10.1051/aas:1999222].

[66] J. Dorschner, B. Begemann, T. Henning, C. Jaeger, and H. Mutschke, "Steps toward interstellar silicate mineralogy. II. Study of Mg-Fe-silicate glasses of variable composition.," Astron. Astrophys. 300, 503-520 (1995). 
[67] C. Jäger, J. Dorschner, H. Mutschke, T. Posch, and T. Henning, "Steps toward interstellar silicate mineralogy. VII. Spectral properties and crystallization behaviour of magnesium silicates produced by the sol-gel method," Astron. Astrophys. 408, 193-204 (2003) [doi: 10.1051/0004-6361:20030916].

[68] D. Fabian, T. Henning, C. Jäger, H. Mutschke, J. Dorschner, and O. Wehrhan, "Steps toward interstellar silicate mineralogy. VI. Dependence of crystalline olivine IR spectra on iron content and particle shape," Astron. Astrophys. 378, 228-238 (2001) [doi: 10.1051/0004-6361:20011196].

[69] H. Sogawa, C. Koike, H. Chihara, H. Suto, S. Tachibana, A. Tsuchiyama, and T. Kozasa, "Infrared reflection spectra of forsterite crystal," Astron. Astrophys. 451, 357-361 (2006) [doi: 10.1051/0004-6361:20041538].

[70] H. Suto, H. Sogawa, S. Tachibana, C. Koike, H. Karoji, A. Tsuchiyama, H. Chihara, K. Mizutani, J. Akedo, K. Ogiso, T. Fukui, and S. Ohara, "Low-temperature single crystal reflection spectra of forsterite," Mon. Not. R. Astron. Soc. 370, 1599-1606 (2006) [doi: 10.1111/j.1365-2966.2006.10594.x].

[71] F. J. Molster, L. B. F. M. Waters, and A. G. G. M. Tielens, "Crystalline silicate dust around evolved stars. II. The crystalline silicate complexes," Astron. Astrophys. 382, 222240 (2002) [doi: 10.1051/0004-6361:20011551].

[72] A. Tamanai, H. Mutschke, J. Blum, and G. Meeus, "The $10 \mu \mathrm{m}$ infrared band of silicate dust: A laboratory study comparing the aerosol and $\mathrm{KBr}$ pellet techniques," Astrophys. J. Lett. 648, L147-L150 (2006) [doi: 10.1086/508164].

[73] A. Tamanai, H. Mutschke, J. Blum, and R. Neuhäuser, "Experimental infrared spectroscopic measurement of light extinction for agglomerate dust grains," J. Quant. Spectrosc. Radiat. Transf. 100, 373-381 (2006) [doi: 10.1016/j.jqsrt.2005.11.051].

[74] A. Tamanai, H. Mutschke, J. Blum, T. Posch, C. Koike, and J. W. Ferguson, "Morphological effects on IR band profiles. Experimental spectroscopic analysis with application to observed spectra of oxygen-rich AGB stars," Astron. Astrophys. 501, 251-267 (2009) [doi: 10.1051/0004-6361/200911614].

[75] A. Tamanai, H. Mutschke, and J. Blum, "IR spectroscopy of free-flying silicate dust grains," in Cosmic Dust - Near and Far, Th. Henning, E. Grün, and J. Steinacker, Eds., Astron. Soc. Pacific Conf. Ser., in press (2009).

[76] J. Bradley, “The astromineralogy of interplanetary dust particles," in Astromineralogy, 2nd rev. ed., Th. Henning, Ed., Springer, Berlin, in press (2010). 